\title{
The Effect of Limbal Mesenchymal Stem Cell Secretome on Corneal Neovascularization in Experimental Model of Corneal Alkali Injury
}

\author{
Farah Azizah $^{1}$, Evelyn Komaratih ${ }^{1}$, Ismi Zuhria ${ }^{1}$ \\ official email address: farahazizah4@gmail.com \\ ${ }^{1}$ Department of Ophthalmology, Faculty of Medicine Universitas Airlangga/ Dr. Soetomo General Academic Hospital, Jl. Mayjen. Prof. \\ Dr. Moestopo 4-7, Surabaya,60286, Indonesia
}

\begin{abstract}
Background: To investigate the effect of limbal mesenchymal stem cell (L-MSC) secretome in inhibition of corneal neovascularization in animal model of alkali chemical injury

Methods: Secretome was collected from limbal mesenchymal stem cells of corneoscleral tissue of healthy male rabbit. Rabbits were exposed to $\mathrm{NaOH}$. The research subjects consisted of 3 groups, normal control group, Balance salt solution (BSS) group, and (L-MSC) secretome group. Clinical corneal neovascularization level, number of corneal neovascularization histopathologically with hematoxylin-eosin staining and VEGF expression by immunohistochemical staining were analyzed.
\end{abstract}

Results: After seven days of administration of topical instillation of L-MSC secretome, neovascularization level in LMSC group was lower than BSS group even though the difference was not statistically significant ( $\mathrm{p}>0.05)$. The mean histopathological neovascular count in the L-MSC secretome group was lower than that BSS group even though the difference was not statistically significant $(\mathrm{p}>0.05)$

Conclusions: In conclusion, topical instillation of limbal mesenchymal stem secretome has a potential effect on inhibiton of corneal neovascularization even though not significant at day-7 in experimental alkali injury model.

Keywords: Limbal mesenchymal stem cell, secretome, corneal neovascularization, corneal alkali injury

\section{Introduction}

Chemical injury is an eye emergency and is one of the leading causes of blindness in the world. Chemical injury of the eye requires immediate and intensive management to reduce severe complications and loss of vision. Alkali agents are the most common cause of chemical injury, which are lipophilic and have the ability to saponify fatty acids from cell membranes, penetrate the corneal stroma, and denature protein structures, causing changes in composition and tissue necrosis. Prompt and appropriate therapy is needed to prevent complications, including slow epithelialization, persistent ulcers, corneal perforation, and angiogenesis due to inflammation, neovascularization, and corneal conjunctivalization. Approximately $11.5 \%-22.1 \%$ of all eye trauma are chemical injuries. Males are more susceptible which is around $73.5 \%$ with the majority of age are 20-40 years. The majority of eye chemical injuries occur due to work accidents in an industrial, which is around 61\% while in the home environment is about 37\% (Badaran-Rafii et al., 2017; Singh et al., 2013; Chen et al., 2020; Mishra et al., 2019). 
In the acute phase of chemical injury, anti-inflammatory, anti-angiogenic therapy, and improvement of corneal epithelial healing are important aspects in the treatment and prognosis. Currently, the use of mesenchymal stem cell (MSC) secretome has been developed for the healing of corneal wounds, including alkali chemical injury. Mesenchymal stem cell secretome has been investigated to have the role as an antiinflammatory, anti-angiogenesis, and immunomodulatory. Several studies have shown that MSC not only reduces inflammation and reduces corneal opacity, but can also significantly inhibits neovascularization in corneal chemical injury (Almaliotis et al., 2015; Cejka et al., 2016; Beeken et al., 2020; Ke et al., 2015; Ma et al., 2006; Pawitan, 2014). The aim of this study is to analyze the effect of limbal mesenchymal stem cell (LMSC) secretome in inhibition of corneal neovascularization in an animal model of alkali chemical injury by evaluating corneal neovascularization clinically and histopathologically.

\section{Material and Method}

\subsection{Limbal mesenchymal stem cell (L-MSCs) secretome}

The cell was obtained from the stem cell laboratory, Universitas Airlangga. To obtain secretome, the amount of $5 \times 103$ cells/well of L-MSC was treated for 24 hours using media composed of $\alpha$-MEM, $1 \%$ amphotericin B, 1\% NEAA, 1\% penicillin-streptomycin, and $2 \%$ FBS). The secretome was collected and filtered using $0.45 \mathrm{um}$ millipore, the osmolarity and acidity were adjusted for 270-300 mOsm/L and 7.2-7.5, respectively. The secretome was packaged into a single dose application in a sterile Eppendorf tube and stored at $-20^{\circ} \mathrm{C}$ until further application.

\subsection{Animal model of alkali injury}

A corneal alkali injury was made in the right eye of each rabbit. The rabbits were anesthetized by intramuscular injection of xylazine-ketamine (ketamine $40 \mathrm{mg} / \mathrm{ml}$, xylazine $20 \mathrm{mg} / \mathrm{ml}$ ). A piece of Whatman filter paper ( $7 \mathrm{~mm}$ diameter) soaked in $1 \mathrm{~N} \mathrm{NaOH}$ was applied to the center of the cornea for 30 seconds. The cornea was then rinsed with $30 \mathrm{ml}$ of saline for 30 seconds.

\subsection{Animal model treatment}

A Total of 21 New Zealand white rabbits were randomly divided into three groups. The first group (normal control group) was the group without $\mathrm{NaOH}$ exposure and therapy. The second group ( $\mathrm{NaOH}+\mathrm{BSS}$ ) was the group with exposure to $\mathrm{NaOH}$ and basal saline solution (BSS) eye drops. The third group (NaOH + limbal MSC secretome) was the group with exposure to $\mathrm{NaOH}$ and limbal MSC secretome eye drops. BSS and limbal MSC secretome eye drops were administered every 8 hours for 7 days. The degree of clinical corneal neovascularization, histopathological neovascular cell count were measured 7 days after treatment.

\subsection{Clinical Evaluation of Corneal Neovascularization}

Clinical evaluation of corneal neovascularization was performed using handheld slit-lamp and captured by digital camera. Peripheral corneal neovascularization was measured from limbus to central using caliper and was scored 0 (no neovascular in the entire cornea); 1 (peripheral neovascularization $<2 \mathrm{~mm}$ ); 2 (peripheral 
neovascularization $>2 \mathrm{~mm}$ but sparing the central cornea); 3 (neovascularization involving the central cornea); 4: (neovascularization involving the central cornea with fibrosis) (Prabhasawat et al., 2012).

\subsection{Histopathology Evaluation of Corneal Neovascularization}

Enucleation was performed and eyeballs were stored in formalin. The histopathology section was sliced in 4 $\mu \mathrm{m}$ and subjected to hematoxylin-eosin staining. Neovascular cell count was observed in 5 fields of view using Nikon H600L microscope under 400x magnification. The result was validated by an anatomic pathologist.

\subsection{Statistical analysis}

The data was analyzed using SPSS version 26.0 where $\mathrm{P}<0.05$ was considered to be statistically significant. Statistical measures are presented in a descriptive table (e.g median, interquartile deviation, mean and standard deviation). Statistical analysis for the degree of corneal neovascularization between 3 groups were analyzed with Kruskal Wallis while between 2 groups were analyzed with Mann Whitney. Statistical analysis for histopathological neovascular count between 3 groups were analyzed with One way ANOVA while between 2 groups were analyzed with posthoc Games Howell.

\section{Results}

\subsection{Clinical corneal neovascularization improvement after limbal mesenchymal stem cell (L-MSCS)} secretome treatment

The results of the study for clinical evaluation showed that there were clinically significant differences in the level of neovascularization in the normal control group, BSS group and the limbal MSC secretome group $(\mathrm{p}=0.001,<0.05)$. There was a clinically significant difference in the level of neovascularization between normal control group and BSS group $(\mathrm{p}=0.001)$ and between normal control group and the limbal MSC secretome group $(\mathrm{p}=0.003)$. The degree of clinical corneal neovascularization was improved in L-MSCs treatment group compared to BSS group though it was not significantly different $(\mathrm{p}=0,100)$ (Table 1 and Figure 1).

Table 1. Degree of clinical corneal neovascularization

\begin{tabular}{|l|c|c|c|c|c|}
\hline \multirow{2}{*}{\multicolumn{1}{c}{ Groups }} & \multirow{2}{*}{$\mathbf{n}$} & \multicolumn{3}{c|}{$\begin{array}{c}\text { Degree of clinical corneal } \\
\text { neovascularization (Scor) }\end{array}$} & \multirow{2}{*}{ P } \\
\cline { 3 - 5 } & & Median \pm IQD & Min & Max & \\
\hline Control & 7 & $0 \pm 0,0$ & 0 & 0 & \multirow{2}{*}{0,001} \\
\hline $\mathrm{NaOH}+\mathrm{BSS}$ & 7 & $2 \pm 0,5$ & 1 & 2 & \\
\cline { 1 - 5 } $\mathrm{NaOH}+\mathrm{L}-\mathrm{MSCs}$ secretome & 7 & $1 \pm 0,5$ & 0 & 2 & \\
\hline
\end{tabular}




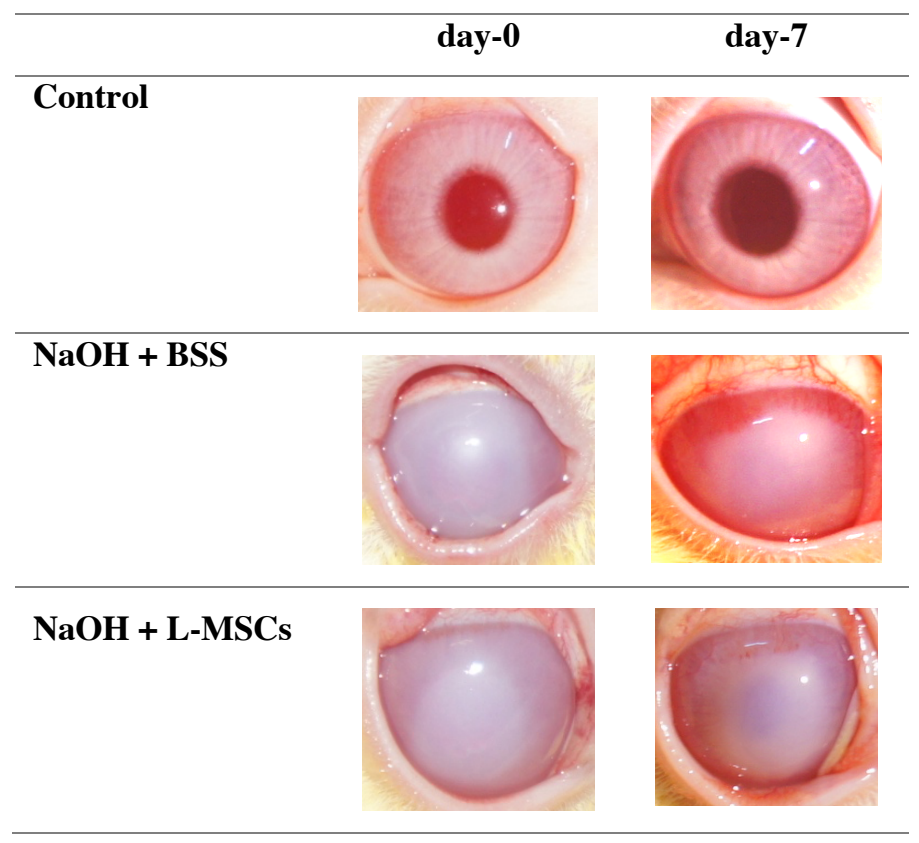

Fig. 1. Clinical evaluation of corneal neovascularization on day-0 and day-7.

3.2. Histopathological corneal neovascularization improvement after limbal mesenchymal stem cell (L-MSCs) secretome treatment

In the histopathological evaluation of the mean neovascular count with hematoxylin-eosin staining, the results in normal control group were $11.1 \pm 3.9$ while limbal MSC secretome group was lower $(42.1 \pm 6.4$ cells/field of view) than BSS group $(66.0 \pm 29.7$ cells/field of view) though their not significantly different $(\mathrm{p}=0.167$, $<0.05)$. There was significant difference in mean neovascular count between normal control group, BSS and limbal MSC secretome group $(p=0.000,<0.05)$. Significant differences were also found between normal control group and BSS group $(\mathrm{p}=0.006)$ and between control group with limbal MSC secretome group $(p=0.000)($ Tabel 1).

Table 1. Degree of clinical corneal neovascularization

\begin{tabular}{|c|c|c|c|c|c|}
\hline \multirow[t]{2}{*}{ Group } & \multirow[t]{2}{*}{$\mathbf{n}$} & \multicolumn{3}{|c|}{$\begin{array}{l}\text { neovascular count } \\
\text { (cells/field of view) }\end{array}$} & \multirow[t]{2}{*}{$\mathbf{P}$} \\
\hline & & Mean \pm SD & Min & Max & \\
\hline Control & 7 & $11,1 \pm 3,9^{\mathrm{a}}$ & 7,6 & 18,2 & \multirow[t]{3}{*}{$0,002^{*}$} \\
\hline $\mathrm{NaOH}+\mathrm{BSS}$ & 7 & $66,0 \pm 29,7^{b}$ & 30,2 & 116,0 & \\
\hline $\mathrm{NaOH}+\mathrm{L}-\mathrm{MSCs}$ & 7 & $42,1 \pm 6,4^{\mathrm{b}}$ & 35,0 & 53,4 & \\
\hline
\end{tabular}


(A)

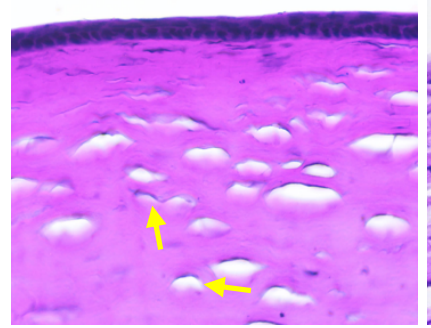

(B)

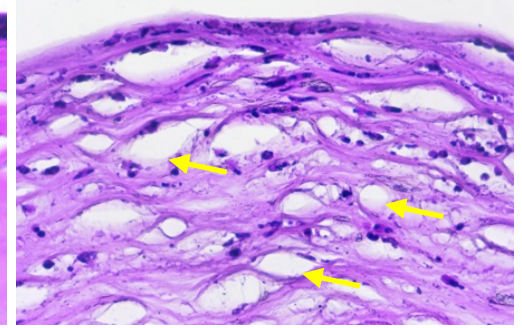

(C)

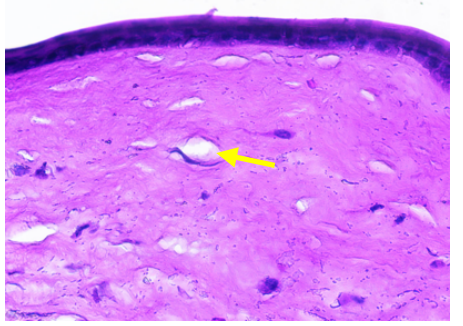

Fig. 2. Histopathological appearance of neovascular cells (yellow arrows) in control group (a) $\mathrm{NaOH}+\mathrm{BSS}(\mathrm{b}) \mathrm{and} \mathrm{NaOH}+\mathrm{L}-\mathrm{MSCs}$ (c). (HE staining; 400x magnification).

\section{Discussion}

Chemical injury is an eye emergency and is one of the leading causes of blindness in the world. Chemical injury of the eye requires immediate and intensive management to reduce severe complications and loss of vision. Alkali agents are the most common cause of chemical injury. In the acute phase of chemical injury, anti-inflammatory, anti-angiogenic therapy and improvement of corneal epithelial healing are important aspects in the treatment and prognosis. Currently, the use of mesenchymal stem cell (MSC) secretome has been developed for healing of corneal wounds, including alkali chemical injury. Mesenchymal stem cell secretome has been investigated to have a role as an anti-inflammatory, antiangiogenesis, and immunomodulatory. Several studies have shown that MSC not only reduces inflammation and reduces corneal opacity, but can also significantly inhibits neovascularization (Oh et al., 2008; Ma et al., 2006; Yao et al., 2012).

In this study, topical instillation of L-MSC secretome inhibits corneal neovascularization even though it is not significant on day-7. Another study supports this result that At 1-week post-injury, the treatment and control group did not show any differences with massive peripheral new vessels in all groups. However, the degree of neovascularization rapidly decreased in the MSC-treated cornea as time passed after the injury. The degree of corneal neovascularization was the lowest in the MSC group and the highest in the control group at three weeks post-injury (Oh et al., 2008).

Histopathologically, neovascular count of L-MSCs treatment group was lower than BSS group even though the difference was not statistically significant on day-7. This represents the potential effect of L-MSCs in inhibiting corneal neovascularization. Another study supports this result that histopathologically, new vessels were marked in the central area of the cornea of the untreated control group, which were absent in the treatment group (injection of bone marrow-derived mesenchymal stem cells (BMSCs) under the conjunctiva) (Ghazaryan et al., 2016).

Corneal neovascularization can occur when there is an imbalance between proangiogenic and antiangiogenic factors which there are excessive pro-angiogenic factors such as vascular endothelial growth factor (VEGF), basic fibroblast growth factor (bFGF) and matrix metalloproteinase (MMP), while there is a deficiency of anti-angiogenic factors such as pigment epithelium-derived factor, angiostatin, endostatin and thrombospondin. MSC inhibit corneal neovascularization by downregulating pro-angiogenic factors (VEGF, MMP) and upregulating anti-angiogenic factors (TSP-1, PEDF, sLFT-1, TSG-6) (Beeken et al., 2020; Eslani et al., 2017; Oh et al., 2008; Ghazaryan et al., 2016; Komaratih et al., 2019; Song et al., 2018; Yao et al., 2012). 
The limitation of this study is that the evaluation was only carried out at one time (day-7), not serially for a longer time. In addition, pro-angiogenic and anti-angiogenic factors were not evaluated. Further studies are mandatory to evaluate in longer time and evaluate angiogenic factors involved.

\section{Conclusion}

In conclusion, topical instillation of limbal mesenchymal stem secretome has a potential effect on inhibition of corneal neovascularization even though not significant at day-7 in experimental alkali injury model.

\section{Acknowledgements}

None.

\section{Conflict of Interest}

Nil.

\section{Ethical Satandard}

Ethical approval was obtained from Institutional Ethical Committee of Faculty of Veterinary medicine, Universitas Airlangga (No: 2.KE.050.05.2021). All Procedures were performed with ethical standards.

\section{References}

Almaliotis, D., G. Koliakos, E. Papakonstantinou. 2015. Mesenchymal Stem Cells Improve Healing Of The Cornea After Alkali Injury. Graefes Arch Clin Exp Ophthalmol. 253(7): 1121-1135.

Baradaran-Rafii, A., M. Eslani, Z. Haq, E. Shirzadeh, M. J. Huvard and A. R. Djalilian. 2017. Current and Upcoming Therapies for Ocular Surface Chemical Injuries. Ocular Surface. 15(1): 48-64.

Beeken, L.J., D.S.J. Ting and L.E. Sidney. 2020. Potential Of Mesenchymal Stem Cells As Topical Immunomodulatory Cell Therapies For Ocular Surface Inflammatory Disorders. Stem Cells Transl Med. 1-12.

Cejka, C., V. Holan, P. Trosan. 2016. The Favorable Effect Of Mesenchy- Mal Stem Cell Treatment On The Antioxidant Protective Mechanism In The Corneal Epithelium And Renewal Of Corneal Optical Properties Changed After Alkali Burns. Oxid Med Cell Longev. 2016: 1-12.

Chen, M., A.A. Gureyee, Y. Cisse and L. Bai. 2020. The Therapeutic Effects and Possible Mechanism of Pranoprofen in Mouse Model of Corneal Alkali Burns. Hindawi Journal of Ophthalmology. 1-9.

Eslani, M., A. Baradaran-Rafii, A. Movahedan and A.R. Djalilian. 2014. The Ocular Surface Chemical Burns. Journal of Ophthalmology. $1-9$.

Ghazaryan, E., Y. Zhang, Y. He, X. Lu, Y. Li, J. Xie and G. Su. 2016. Mesenchymal stem cells in corneal neovascularization: Comparison of different application routes. Molecular Medicine Reports. 14: 3104-3112.

Ke, Y., Y. Wu, X. Cui, X. Liu, M. Yu, C. Yang \& X. Li. 2015. Polysaccharide Hydrogel Combined With Mesenchymal Stem Cells Promotes The Healing Of Corneal Alkali Burn In Rats. PLoS One. 10(3): 
$1-18$.

Komaratih, E., Y, Rindiastuti, Y.W. Wirohadidjojo, D. Lutfi, Nurwasis, F.A. Rantam, N. Ertanti \& C.R.S. Prakoeswa. 2019. A Comparative Study on The Therapeutic Potential of Ocular and Non-Ocular Stem Cell Secretome on Alkali-Induced Limbal Stem Cell Niche Damage. Biochem. Cell. Arch. 19(2): 4825-4832.

Ma, Y., Y. Xu, Z. Xiao, W. Yang, C. Zhang, E. Song, Y. Du \& L. Li. 2006. Reconstruction of Chemically Burned Rat Corneal Surface by Bone Marrow-Derived Human Mesenchymal Stem Cells. Stem Cells. 24: 315-321.

Mishra, B.P., A. Mahapatra, S.K. Sahu, C. Naik and S.S. Dany. 2019. Incidence and Management of Chemical Injuries of Eye. Journal of Medical Science and Clinical Research. 7(06): 807-812.

Oh JY, Kim MK, Shin MS, et al. 2008. The Anti-Inflammatory And Anti- Angiogenic Role Of Mesenchymal Stem Cells In Corneal Wound Healing Following Chemical Injury. STEM CELLS. 26(4):1047-1055.

Pawitan, J.A. 2014. Prospect of Stem Cell Conditioned Medium in Regenerative Medicine. BioMed Research International. 14: 1-14.

Prabhasawat, P., Ekpo, P., Uiprasertkul, M., Chotikavanich, S., Tesavibul, N., Pornpanich, K., \& Luemsamran, P. 2016. Long-Term Result Of Autologous Cultivated Oral Mucosal Epithelial Transplantation For Severe Ocular Surface Disease. Cell And Tissue Banking. 17(3): 491-503.

Singh, M.P., Y. Tyagi, K. Kumar, Gupta and P. Sharma. 2013. Ocular Chemical Injuries and Their Management. Oman Journal of Ophthalmology. 6(2): 83.

Song HB, Park SY, Ko JH. 2018. Mesenchymal stromal cells inhibit inflammatory lymphangiogenesis in the cornea by suppressing macrophage in a TSG-6-dependent manner. Mol Ther. 26(1): 162-172.

Yao, L., Z.R. Li, W.R. Su, Y.P. Li, M.L. Lin, W.X. Zhang, Y. Liu, Q. Wan and D. Liang. 2012. Role of Mesenchymal Stem Cells on Cornea Wound Healing Induced by Acute Alkali Burn. Plos One. 7(2): $1-7$.

Ye J, Yao K, Kim JC. 2006. Mesenchymal stem cell transplantation in a rabbit corneal alkali burn model: engraftment and involvement in wound healing. Eye (Lond) 20: 482-490. 